\title{
Antitumor activity of a novel small molecule STAT3 inhibitor against a human lymphoma cell line with high STAT3 activation
}

\author{
TADASHI ASHIZAWA ${ }^{1}$, HARUO MIYATA ${ }^{1}$, HIDEE ISHII ${ }^{1}$, CHIE OSHITA $^{1}$, KENJI MATSUNO ${ }^{2}$, \\ YOSHIAKI MASUDA ${ }^{3}$, TOSHIO FURUYA ${ }^{3}$, TADASHI OKAWARA ${ }^{4}$, MASAMI OTSUKA $^{5}$, \\ NAOHISA OGO $^{6}$, AKIRA ASAI ${ }^{2}$ and YASUTO AKIYAMA ${ }^{1}$ \\ ${ }^{1}$ Immunotherapy Division, Shizuoka Cancer Center Research Institute, Nagaizumi-cho, Sunto-gun, Shizuoka 411-8777; \\ ${ }^{2}$ Graduate School of Pharmaceutical Sciences, University of Shizuoka, Suruga-ku, Shizuoka 422-8526; \\ ${ }^{3}$ PharmaDesign, Inc., Chuo-ku, Tokyo 104-0032; ${ }^{4}$ Kumamoto Health Science University, Kumamoto 861-5598; \\ ${ }^{5}$ Faculty of Medical and Pharmaceutical Sciences, Kumamoto University, Kumamoto 862-0973; \\ ${ }^{6}$ Shizuoka Institute of Environment and Hygiene, Aoi-ku, Shizuoka 420-8637, Japan
}

Received November 30, 2010; Accepted January 7, 2011

DOI: 10.3892/ijo.2011.957

\begin{abstract}
Signal transducer and activator of transcription (STAT)3, a member of a family of DNA-binding molecules mediating numerous physiological and oncogenic signaling pathways, is a novel target in cancer cells which show high phosphorylation of STAT3. Recently, we identified a novel small-molecule inhibitor of STAT3 dimerization, STX-0119, as a cancer therapeutic. We investigated the mechanisms responsible for the antitumor activity in vitro and in vivo through numerous biochemical and biological assays. Specifically, the effects of STX-0119 on target genes (c-myc, cyclin D1, survivin) and apoptosis induction were analyzed in tumors treated with STX-0119 in vivo. STX-0119 showed strong growth-inhibitory activity against a broad range of hematological cancer cell lines, particularly lymphomas. STX-0119 suppressed the growth of SCC 3 cells, a human lymphoma cell line with highly activated STAT3, through apoptosis and down-regulation of STAT3 targets such as c-myc, cyclin D1, survivin and Bcl-xL. Notably, Tyr-705-phosphorylated STAT3 up-regulation was not significantly suppressed by STX-0119, as opposed to other STAT3 inhibitors. STX-0119 demonstrated potent antitumor effects in vivo in SCC3-bearing nude mice by way of the down-regulation of STAT3 target genes
\end{abstract}

Correspondence to: Dr Yasuto Akiyama, Immunotherapy Division, Shizuoka Cancer Center Research Institute, 1007 Shimonagakubo, Nagaizumi-cho, Sunto-gun, Shizuoka 411-8777, Japan

E-mail: y.akiyama@scchr.jp

Abbreviations: STAT, signal transducer and activator of transcription; SH, Src homology; DMSO, dimethyl sulfoxide; JAK, Janus kinase; T/C, tumor/control; siRNA, small interfering RNA; shRNA, small hairpin RNA

Key words: STAT3, dimerization, phosphorylated STAT3, lymphoma and induction of apoptosis in the tumors. Thus, STX-0119 may be a new type of STAT3 inhibitor exhibiting strong antitumor activity.

\section{Introduction}

The signal transducer and activator of transcription (STAT) protein family is a group of transcription factors that play an important role in relaying signals from growth factors and cytokines (1-3). STAT3 is reported to be involved in oncogenesis by up-regulating the transcription of several genes that control tumor cell survival, resistance to apoptosis, cell cycle progression and angiogenesis. Targets of STAT3 include Bcl-2 (4), Bcl-xL (5), c-myc (6), cyclin D1 (7), vascular endothelial growth factor (8) and human telomerase reverse transcriptase (9).

Several STATs have been associated with the proliferation and survival of cancer cells; however STAT3 is also widely expressed in normal cells. Generally, STAT3 is temporarily activated in a manner strictly regulated by the protein inhibitor of STATs (PIAS), Src homology (SH)2-containing tyrosine phosphatases (SHP1 and SHP2) (10), and suppressor of cytokine signaling/extracellular signal-regulated kinase (SOCS/ERK) (11) to protect cells from excess inflammatory signaling.

Moreover, persistent activation of STAT3 occurs in many hematological malignancies $(12,13)$ and solid cancers. Constitutive STAT3 activation up-regulates the expression of anti-apoptotic factors including Bcl-xL, Bcl-2 and Mcl-1 and promotes the survival of tumor cells, thus contributing to resistance to FAS- or chemotherapy-induced apoptosis (14). Blocking of STAT3 signaling demonstrated significant growth inhibition of cancer cells by down-regulation of the expression of anti-apoptotic genes, leading to apoptosis.

The activation of STAT3 occurs when it is phosphorylated at the Tyr-705 residue. This leads to dimerization and a subsequent transfer to the nucleus where further activation promotes the binding of DNA and the translation of target genes. We are currently designing inhibitors of STAT3 
dimerization by simulating docking in silico and by screening for active compounds through biochemical methods and with in vitro as well as in vivo systems (15).

In the present study we demonstrated the antitumor activity of a novel STAT3-SH2 antagonist, STX-0119, against a constitutively STAT3-activated human lymphoma cell line and investigated the mechanism responsible for the efficacy of STX-0119.

\section{Materials and methods}

Cell lines and reagents. All hematological cancer cell lines including IM-9, U937, Jurkat, KG-1, MOLT-3, HL-60, THP-1, RPMI8226, HUT78, HH, K562, BC-3, U266B1, NCI-H929 and TF-1 were purchased from American Type Culture Collection (Manassas, VA, USA). SCC3 was supplied by Health Science Research Resources Bank (Osaka, Japan). The CMK cell line was kindly provided by Dr T. Sato of Chiba University School of Medicine. All cell lines were cultured in RPMI1640 medium (Sigma-Aldrich, St. Louis, MO) supplemented with L-glutamine $(2 \mathrm{mM})$, penicillin $(100 \mathrm{U} / \mathrm{ml})$, streptomycin (100 U/ml) and 10\% (v/v) fetal bovine serum (FBS; Gibco, Paisley, UK). Various antibodies against STAT3, phosphospecific STAT3 (Tyr-705), c-myc, cyclin D1, survivin, Bcl-xL and $\beta$-actin were purchased from Cell Signaling Technology, Inc. (Danvers, MA) and Becton-Dickinson (BD) Biosciences (Franklin Lakes, NJ) for Western blotting (WB). Anti-STAT3 and anti-phospho-specific STAT3 polyclonal antibodies (Cell Signaling Technology), anti-Ki76 antibody (clone MIB-1; Dako, Glostrup, Denmark) and anti-c-myc antibody (Abcam, Cambridge, MA) were purchased for immunohistochemistry (IHC). Phycoerythin (PE)-labeled mouse anti-phosphorylated STAT3 monoclonal antibody (mAb) was purchased from BD Biosciences for FACS analysis.

Chemicals. STX-0119 and the JAK-specific inhibitor AG490 were synthesized and supplied by the Center for Drug Discovery, University of Shizuoka, Shizuoka, Japan and by Dr Okawara, Kumamoto Health Science University, Kumamoto, Japan, respectively. Cytarabine (Ara-C) was purchased from Wako Pure Chemical Industries, Ltd., Osaka, Japan. These compounds were dissolved and diluted in a sterile $0.5 \%$ (w/v) methylcellulose $400 \mathrm{cp}$ solution (Wako, Tokyo, Japan) or sterile $0.9 \% \mathrm{NaCl}$ solution (Ohtsuka Pharmaceutical, Tokyo, Japan) just prior to their use. The chemical structure of STX-0119 is shown in Fig. 1.

Cell proliferation assay. Cell proliferation was determined using the WST-1 assay (Dojin Kagaku Corp., Japan). A $100 \mu 1$ volume of floating cells $\left(3 \times 10^{4}\right.$ to $\left.1 \times 10^{5} / \mathrm{ml}\right)$ was seeded into each well of a 96-well microculture plate (Corning, NY), and $100 \mu \mathrm{l}$ of various doses of compounds sequentially diluted with DMSO $(100-0.1 \mu \mathrm{M})$ was added. After $72 \mathrm{~h}$ at $37^{\circ} \mathrm{C}$, $10 \mu \mathrm{l}$ of combined WST-1 substrate was added to each well and incubated for $3 \mathrm{~h}$ at $37^{\circ} \mathrm{C}$. The optical density (OD) was measured at 450 and $620 \mathrm{~nm}$ using an immunoreader (Immuno Mini NJ-2300; Nalge Nunc International, Roskilde, Denmark). The $\mathrm{IC}_{50}$ value was defined as the dose needed for a $50 \%$ reduction in OD calculated from the survival curve. Percent survival was calculated as follows: (Mean OD of the

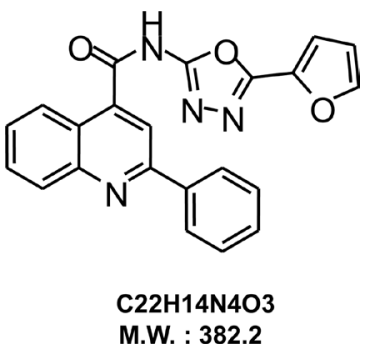

Figure 1. Chemical structure of STX-0119. Formula, $\mathrm{C}_{22} \mathrm{H}_{14} \mathrm{~N}_{4} \mathrm{O}_{3}$. Molecular weight, 382.2.

test wells - Mean OD of the background wells)/(Mean OD of the control wells - Mean OD of the background wells).

STAT3 phosphorylation assay. Hematological cancer cell lines were treated with dimethyl sulfoxide (DMSO; SigmaAldrich), STX-0119 or AG490 overnight in RPMI1640 medium containing $10 \%$ FBS. After being washed, cells were incubated in serum-free RPMI1640 medium for 1-2 h and stimulated with $50 \mathrm{ng} / \mathrm{ml}$ of human interleukin (IL)-6 (Peprotech Biotech, Rocky Hill, NJ). Cells were fixed with fixation buffer (BD Bioscience), permealized with Perm buffer (BD Bioscience) and washed with staining buffer (BD Bioscience). Treated cells were stained with a PE-labeled antiphospho-specific STAT3 antibody or isotype control antibody. Stained cells were fixed with $0.5 \%$ paraformaldehyde (SigmaAldrich) and analyzed on a flow cytometer (FACSCanto, BD).

Apoptosis assay. Cancer cell lines were double-stained with Annexin V and 7-amino-actinomycin D (7-AAD) using an Annexin V-PE apoptosis detection kit (BD Biosciences) according to the manufacturer's instructions. Briefly, after incubation with STX-0119 or AG490 for $24 \mathrm{~h}$, cells were re-suspended in $1 \mathrm{X}$ binding buffer at a concentration of $1 \times 10^{6}$ cells $/ \mathrm{ml}$ and incubated with Annexin V-PE and 7-AAD solutions at room temperature (RT) for $15 \mathrm{~min}$. The percentage of apoptotic cells was analyzed on a flow cytometer (FACSCanto). Early apoptotic cells were defined as Annexin $\mathrm{V}^{+} / 7-\mathrm{AAD}^{-}$.

Western blotting. SCC3 cells were treated with STX-0119, AG490 or DMSO for $24 \mathrm{~h}$ in RPMI1640 medium containing $10 \%$ FBS. Cells were lysed using RIPA buffer (Thermo Fisher Scientific Inc., Rockford, IL) containing protease inhibitors and phosphatase inhibitors. RIPA buffer consisted of $50 \mathrm{mM}$ HEPES (pH 7.4), $150 \mathrm{mM} \mathrm{NaCl}, 1 \mathrm{mM}$ EDTA, $1.5 \mathrm{mM}$ $\mathrm{MgCl}_{2}, 100 \mathrm{mM} \mathrm{NaF}, 10 \mathrm{mM} \mathrm{Na} \mathrm{P}_{2} \mathrm{O}_{7} \cdot 10 \mathrm{H}_{2} \mathrm{O}, 1 \mathrm{mM} \mathrm{NaVO}$, $10 \%$ glycerol and 1\% NP-40. The cell lysate was subjected to SDS-PAGE using a $7.5 \%$ polyacrylamide separating gel, and was subsequently transferred to PVDF membranes. The membranes were blocked with $5 \%$ skim milk in TBS containing $0.1 \%$ Tween-20 (TBS-T) at RT for $60 \mathrm{~min}$ or $4^{\circ} \mathrm{C}$ overnight, and incubated at $4^{\circ} \mathrm{C}$ overnight with the primary antibody against STAT3, phospho-specific STAT3, cleaved caspase-3, c-myc, cyclin D1, Bcl-xL, survivin and $\beta$-actin (1:200-1:2000) in blocking solution.

After washing in TBS-T, the membranes were incubated for $1 \mathrm{~h}$ with horseradish peroxidase (HRP)-conjugated anti-mouse IgG (1:5000). Membranes were treated with 
ECL plus reagent (GE Healthcare) and analyzed on a chemiluminescence scanner (LAS-3000; FujiFilm, Tokyo). Subcellular fractionation analysis of phosphorylated STAT3 in the STX-0119-treated SCC3 cells was also performed using a Subcellular Proteome Extraction kit (Merck kGaA, Darmstadt, Germany). Each subcellular fraction derived from the SCC3 cells was analyzed by WB. In some experiments, SCC3 tumors transplanted into nude mice and treated with STX-0119 were resected on day 3, 7, 14 and 21 after the start of STX-0119 administration and used for WB. WB signals obtained by a chemiluminescence scanner were transferred to a computer-associated image analyzer (WinRoof, version 6; Mitani Corporation, Fukui, Japan). The entire area of each signal was measured and utilized for quantitative analysis.

Cell cycle analysis. Cancer cell lines were incubated with STX-0119 for $24 \mathrm{~h}$, washed with phosphate-buffered saline (PBS) and fixed with ice-cold 70\% (vol/vol) ethanol. After incubation in PBS containing $0.25 \mathrm{mg} / \mathrm{ml}$ of RNase A (type 1-A; Sigma-Aldrich) and $0.1 \%$ (vol/vol) Nonidet P-40 (Nacalai Tesque, Kyoto, Japan) for $30 \mathrm{~min}$ at $37^{\circ} \mathrm{C}$, the cells were stained with $50 \mu \mathrm{g} / \mathrm{ml}$ of propidium iodide (PI; Sigma-Aldrich) for at least $20 \mathrm{~min}$ on ice. Finally, the DNA content was analyzed with a flow cytometer (FACSCalibur, BD) using ModFit LT ${ }^{\mathrm{TM}}$ 3.1 (Verity Software House, Inc., Topsham, ME).

Animal experiments. Male nude mice (BALB/cA-nu/nu, 5-6 weeks old) were obtained from Nippon Clea (Tokyo, Japan), housed in a separate experimental room, and given sterilized food and water ad libitum. All animals were cared for and used humanely according to Guidelines for the Welfare and Use of Animals in Cancer Research (Committee of the National Cancer Research Institute, 2010) and procedures approved by the Animal Care and Use Committee of Shizuoka Cancer Center Research Institute.

SCC3 cells re-suspended in RPMI1640 medium (100 $\mu \mathrm{l})$ containing Matrigel (BD Biosciences) at $1 \times 10^{7} / \mathrm{ml}$, were inoculated into the flank of BALB/cA-nu/nu mice. To evaluate the antitumor activity against subcutaneously (s.c.) inoculated tumors, tumor volume was calculated based on the National Cancer Institute formula as follows: Tumor volume $\left(\mathrm{mm}^{3}\right)=$ Length $(\mathrm{mm}) \times$ [Width $(\mathrm{mm})]^{2} \times 1 / 2$.

The efficacy of the compounds against the human tumors inoculated into the nude mice was expressed as the mean $\mathrm{V} / \mathrm{V}_{0}$ value, where $\mathrm{V}$ is the tumor volume on the day of evaluation and $\mathrm{V}_{0}$ is that on the day of treatment. The tumor/control $(\mathrm{T} / \mathrm{C})$ value was calculated as the mean $\mathrm{V} / \mathrm{V}_{0}$ value of the treated group versus that of the untreated group.

In another experiment, SCC3 tumors treated with vehicle or STX-0119 were resected on day 3, 714 and 21 after the start of STX-0119 administration.

Immunohistochemistry (IHC). SCC3 tumors treated with vehicle or STX-0119 were resected on day 3, 7, 14 and 21 after the start of STX-0119 administration and fixed with formalin solution. Hematoxylin and eosin staining was performed according to the manufacturer's instructions. For immunostaining, antibodies against STAT3, phospho-specific STAT3, c-myc and Ki67 as primary antibody and goat or rabbit anti-mouse IgG antibody as secondary antibody were used.
Table I. Cell proliferation assay using hematological cancer cell lines.

\begin{tabular}{lrr}
\hline & \multicolumn{2}{c}{ IC $_{50}$} \\
\cline { 2 - 3 } Cell line & STX-0119 $(\mu \mathrm{M})$ & AG490 $(\mu \mathrm{M})$ \\
\hline IM-9 & 5.1 & 2.2 \\
U937 & 7.7 & 31.5 \\
Jurkat & 5.3 & 4.2 \\
KG-1 & 6.1 & 15.6 \\
MOLT-3 & 4.6 & 3.2 \\
HL-60 & 17.2 & 86.3 \\
THP-1 & 13.5 & 37.7 \\
RPMI8226 & 8.8 & 19.7 \\
SCC3 & 5.5 & 16.4 \\
HUT78 & 8.2 & 3.5 \\
HH & 1.4 & 4.9 \\
K562 & 18.0 & 50.9 \\
CMK & 7.4 & 7.4 \\
BC-3 & 18.3 & 23.1 \\
U266B1 & $>100$ \\
NCI-H929 & 73.0 & 14.9 \\
TF-1 & 16.3 & 33.6 \\
\hline
\end{tabular}

HRP and hydrogen peroxide were utilized for producing color according to the manufacturer's instructions (Histofine mousestain kit; Nichirei Biosciences Inc., Tokyo).

Statistical analysis of tumor models. Statistical analysis was performed with corrected p-values for comparison with the untreated control using the Steel multiple plus Kruskal-Wallis method and Mann-Whitney's rank-sum test.

\section{Results}

Cell proliferation assay. STX-0119 exhibited a potent antiproliferative effect, with an $\mathrm{IC}_{50}$ of 5-10 $\mu \mathrm{M}$, on hematological cancer cells, particularly acute lymphocytic leukemia (ALL), T-cell lymphoma and myeloma cell lines (Table I). Acute myelocytic leukemia (AML) and chronic myelocytic leukemia (CML) cell lines were not very sensitive. Notably, the T-cell lymphoma cell line $\mathrm{HH}$ was the most sensitive $\left(\mathrm{IC}_{50}, 1.4 \mu \mathrm{M}\right)$ among the 17 hematological cancer cell lines. Moreover, the JAK2 kinase inhibitor, AG490, was not effective against T-cell lymphoma cell lines such as U937, SCC3 and HH when compared with STX-0119.

STAT3 phosphorylation assay. The activation (phosphorylation) of STAT3 was investigated in 10 hematological cell lines with or without IL-6 treatment (Fig. 2A). The phosphorylation was promoted in UP37, KG-1, RPMI8226, THP-1 and CMK cells by IL- 6 or leukemia inhibitory factor (LIF). In contrast, SCC3 which had the strongest STAT3 activation, was demonstrated to be constitutively STAT3-autophosphorylated.

Effect of STX-0119 on phosphorylated STAT3 expression. The effect of STX-0119 on phosphorylated STAT3 expression in the SCC3 and U937 lymphoma cell lines was investigated 


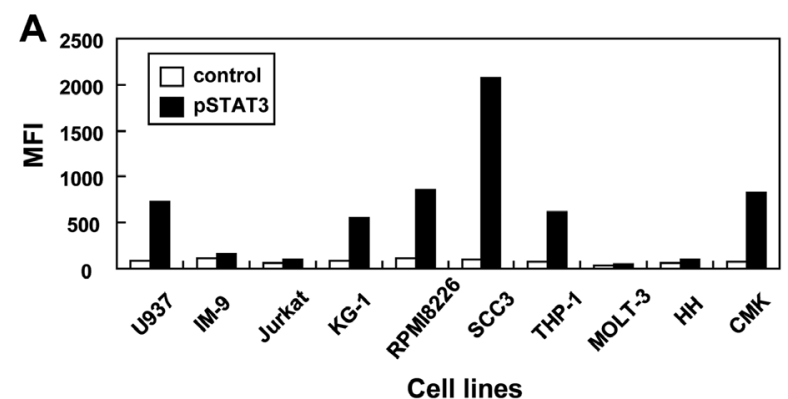

B
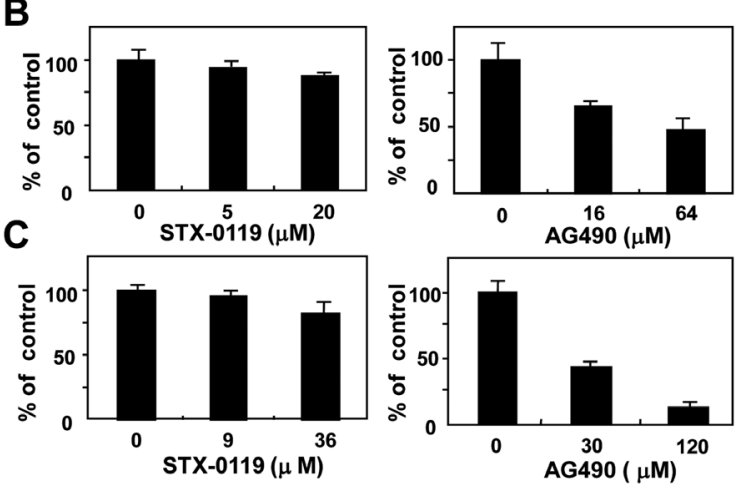

Figure 2. Effect of STX-0119 on STAT3 phosphorylation. (A) STAT3 activation status in hematological cancer cell lines. STAT3 was activated by IL-6 in U937, KG-1, RPMI8226, THP-1 and by LIF in CMK cells. SCC3 cells showed high constitutive STAT3 activation without IL-6 stimulation. After SCC3 (B) or U937 (C) cells were treated with STX-0119 or AG490 overnight, they were stimulated with IL-6 and subsequently stained with anti-phospho-specific STAT3 Ab.
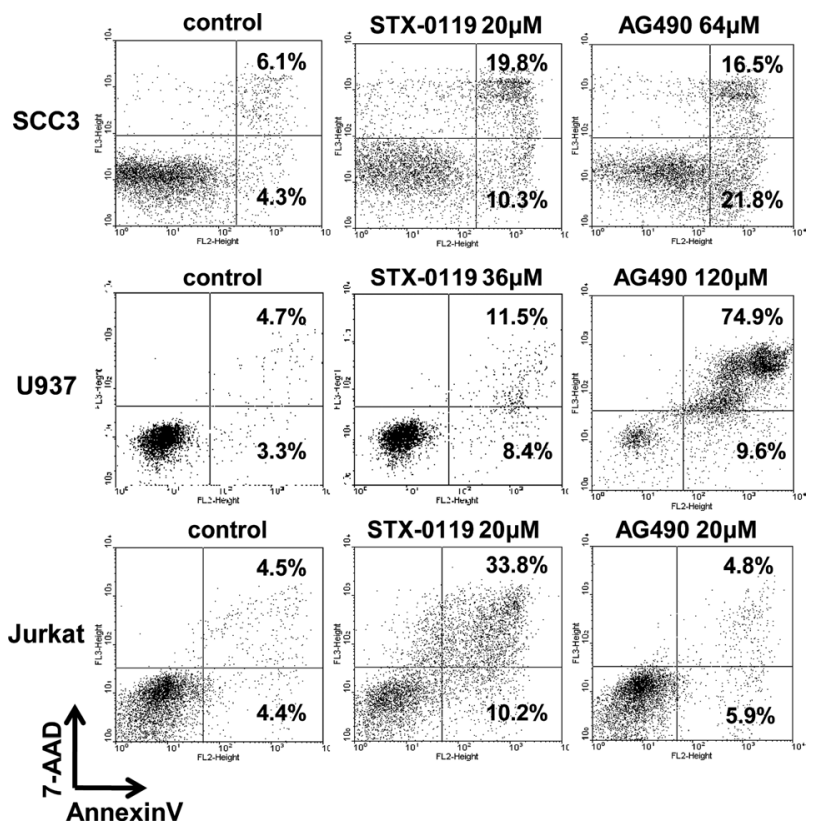

Figure 3. Apoptosis-inducing activity of STX-0119. After incubation with STX-0119 or AG490 for $24 \mathrm{~h}$, SCC3, U937 and Jurkat cells were stained with Annexin V-PE and 7-AAD solutions. STX-0119 showed a weak to moderate apoptosis-inducing activity in the SCC3 and U937 cells while AG490 exhibited a stronger effect than STX-0119.

using flow cytometry. The cells were treated with the $\mathrm{IC}_{50}$ and 4-fold the $\mathrm{IC}_{50}$ of STX-0119 or AG490 overnight and stained with an anti-phospho-specific STAT3 Ab. No significant
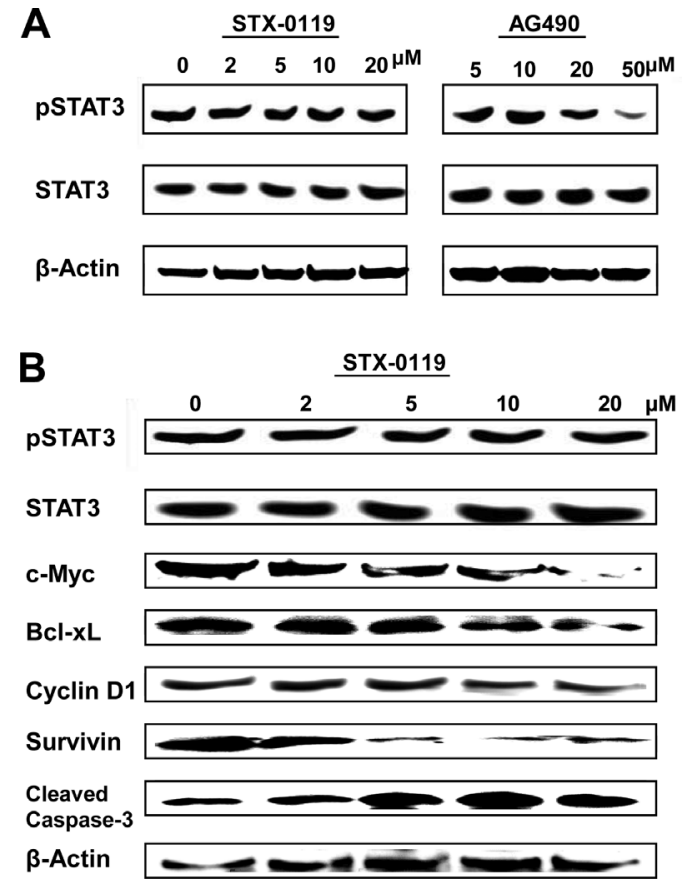

Figure 4. Down-regulation by STX-0119 of the expression of genes targeted by STAT3 in the SCC3 cells. The lysate of SCC3 cells treated with STX-0119, AG490 or DMSO for $24 \mathrm{~h}$ was subjected to WB analysis of target gene expression. Downstream target genes including Bcl-X1, c-myc, cyclin D1 and survivin were significantly down-regulated in their expression by STX-0119. In contrast, an increase in cleaved caspase-3 expression was noted. (A) STAT3 and phosphorylated STAT3 expression, (B) STAT3 downstream target gene expression.

effect of STX-0119 at any dose was noted (Fig. 2B and C) in either the SCC3 or U937 cells. By contrast, AG490 suppressed phosphorylated STAT3 levels in both cell lines in a dosedependent manner.

Induction of apoptosis by STX-0119 in hematological cancer cell lines. The cell lines SCC3 (STAT3 constitutively activated), U937 (STAT3 activated by IL-6) and Jurkat (STAT3 unactivated) were used for the apoptosis assays. STX-0119 showed weak to moderate apoptosis-inducing activity in the SCC3 and U937 cells; however AG490 showed a stronger effect on Annexin ${ }^{+}$apoptotic cells, particularly U937 cells (Fig. 3). Notably, STX-0119 exhibited far stronger apoptosisinducing activity in Jurkat cells compared to AG490.

Regulation of STAT3 target gene expression by STX-0119 in SCC3 cells. Downstream targets including Bcl-xL, c-myc, cyclin D1 and survivin were significantly down-regulated by STX-0119 at $\sim 10 \mu \mathrm{M}$ (Fig. 4). Meanwhile, an increase in cleaved caspase-3 was noted, consistent with the evidence of apoptosis shown previously. In contrast, the expression of STAT3 and phosphorylated STAT3 was not significantly affected by STX-0119, although AG490 strongly suppressed STAT3 phosphorylation in the SCC3 cells.

Effect of STX-0119 on the amount of phosphorylated STAT3 in subcellular fractions. To localize Tyr-705-phosphorylated STAT3 in SCC3 cells treated with STX-0119, subcellular extraction was performed using a Subcellular Proteome Extraction kit. Extracted fractions consisted of cytosolic, 


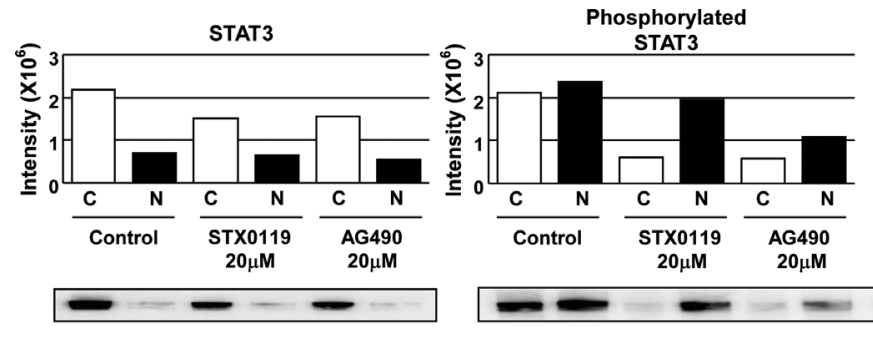

C; cytosol, N; nucleus

Figure 5. Subcellular localization of phosphorylated STAT3 in STX-0119treated SCC 3 cells. SCC 3 cells treated with DMSO (control), STX-0119 $(20 \mu \mathrm{M})$ and AG490 $(20 \mu \mathrm{M})$ were subjected to subcellular fractionation and WB. C, cytosol; N, nucleus. Membrane and cytoskeletal fractions are not shown. STAT3 was not detected in these fractions.

membrane, nuclear and cytoskeletal fractions. Total STAT3 was located mainly in the cytosol and membrane fractions. In contrast, phosphorylated STAT3 accumulated in the nuclear fraction. STX-0119 decreased the amount of phosphorylated STAT3 in the cytosol, but not in the nuclear fraction. However, phosphorylated STAT3 levels in the whole lysate appeared to be similar even after STX-0119 treatment. In contrast, AG490 significantly decreased the amount of phosphorylated STAT3 in both the cytosol and nuclear fractions (Fig. 5).

Effect of STX-0119 on the cell cycle. After incubation with $20 \mu \mathrm{M}$ STX-0119 or $20 \mu \mathrm{M}$ AG490, SCC3 cells were stained with PI, and the DNA content was analyzed with a flow cytometer. STX-0119 had no significant effect on the cell cycle of SCC3 cells compared to the untreated cells (data not shown). Similarly, AG490 showed no obvious effect on the cells at concentrations up to $20 \mu \mathrm{M}$.

STX-0119 inhibits tumor growth in a subcutaneous model of lymphoma. To determine whether STX-0119 inhibits the growth of human tumors, nude mice were transplanted with SCC 3 human lymphoma cells. STX-0119 at doses of 40 and $160 \mathrm{mg} / \mathrm{kg}$ suspended with methylcellulose was orally administered to SCC3-bearing nude mice for five consecutive days. Cytarabine (Ara-C) at $13 \mathrm{mg} / \mathrm{kg}$ as a reference was intraperitoneally administered for 5 consecutive days. In some experiments, AG490 at $25 \mathrm{mg} / \mathrm{kg}$ was administered intraperitoneally to compare its activity to STX-0119 at $20 \mathrm{mg} / \mathrm{kg}$. STX-0119 at more than $80 \mathrm{mg} / \mathrm{kg}$ caused a more than $50 \%$ inhibition of SCC 3 tumor growth on day 4 (early phase) similar to Ara-C (Fig. 6). STX-0119 at $160 \mathrm{mg} / \mathrm{kg}$ alone maintained the growth inhibition at $\sim 50 \%$ as the $\mathrm{T} / \mathrm{C}$ ratio even after day 21 (late phase), although rebound growth was noted in other groups. In the in vivo experiments using SCC3-bearing nude mice, AG490 at $25 \mathrm{mg} / \mathrm{kg}$ revealed a less potent antitumor activity than STX-0119 at $20 \mathrm{mg} / \mathrm{kg}$ (Fig. 6D).

Additionally, no significant side effects including weight loss were noted in the STX-0119-treated mice (Fig. 6C). A brief toxicity test with $160 \mathrm{mg}$ of STX-0119 administered orally for 5 consecutive days showed no changes in hematological or biochemical parameters (data not shown).

Regulation of STAT3 target gene expression by STX-0119 in the SCC 3 tumors. The SCC 3 tumors treated with vehicle, 80 or $160 \mathrm{mg} / \mathrm{kg}$ of STX-0119 were resected on day 3, 7, 14 and 21 and subjected to WB analysis for STAT3 target gene expression. The
A

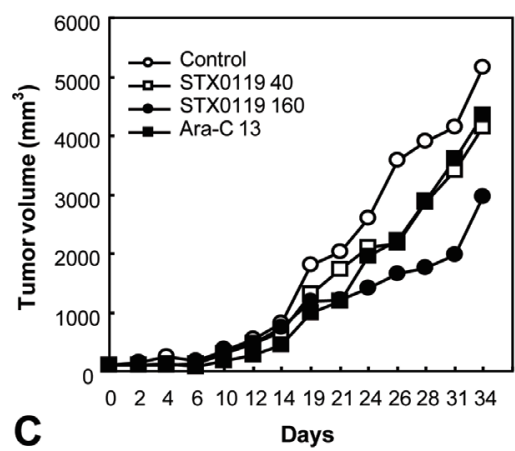

C

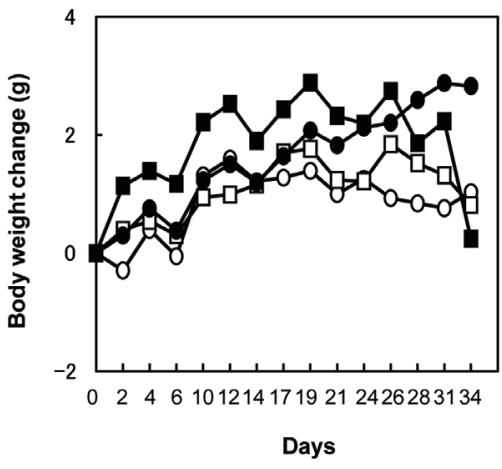

B
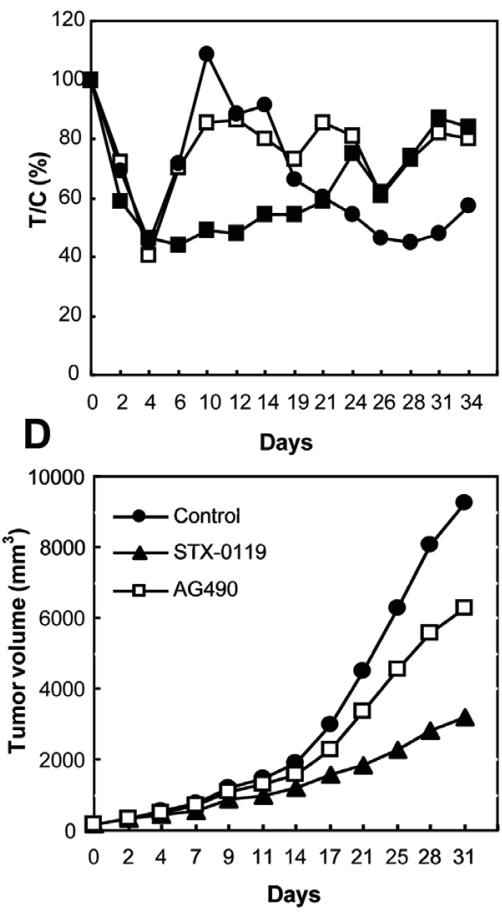

Figure 6. STX-0119 inhibits tumor growth in a subcutaneous model of human lymphoma. Nude mice were transplanted with SCC3 human lymphoma cells. STX-0119 and Ara-C were administered and (A) tumor volume, (B) tumor/control (T/C) ratio and (C) body weight change were assessed. O, control; $\square$, STX0119 (40 mg/kg); •, STX-0119 (160 mg/kg); , Ara-C (13 mg/kg). STX-0119 (20 mg/kg) and AG490 (25 mg/kg) were administered and (D) tumor volume was assessed. •, control; ^, STX-0119 (20 mg/kg); $\square$, AG490 (25 mg/kg). 

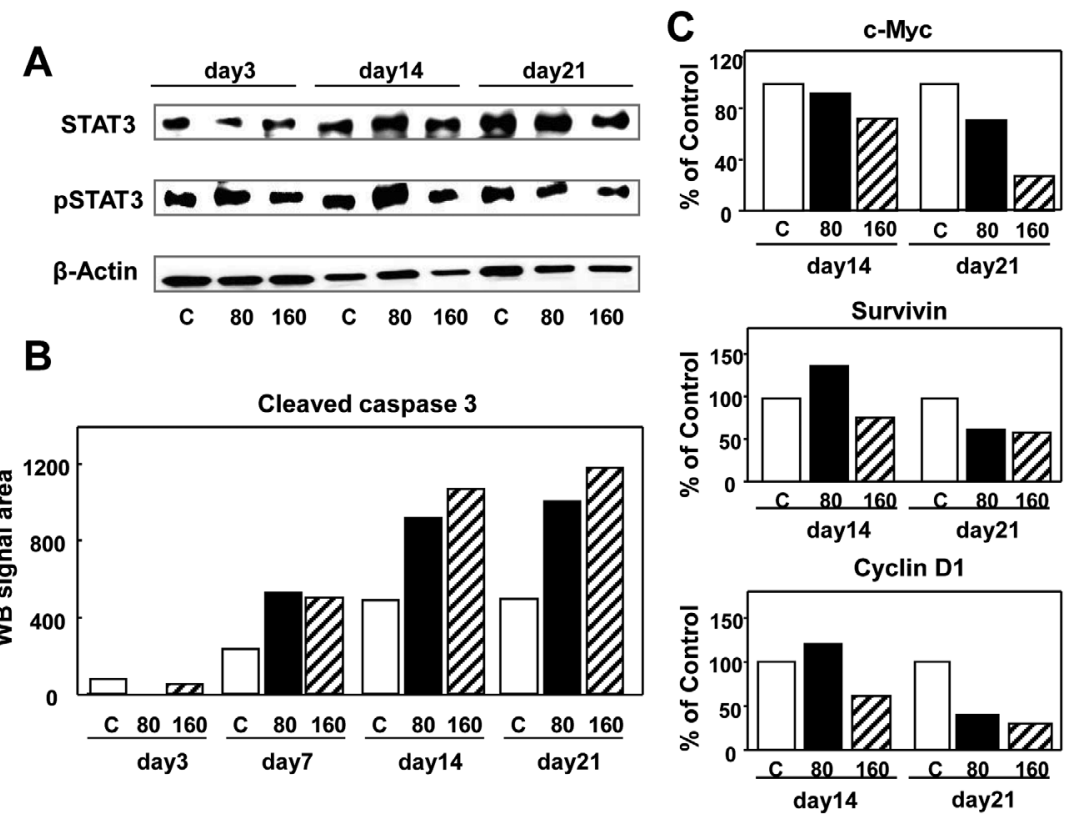

Figure 7. Regulation of STAT3 target gene expression by STX-0119 in SCC3 in vivo tumors. SCC3 tumors treated with 80 and $160 \mathrm{mg} / \mathrm{kg}$ of STX-0119 were resected on day 3, 7, 14 and 21 after the start of administration and were subjected to WB analysis for target gene expression. Expression of (A) STAT3 and phosphorylated STAT3; (B) cleaved caspase 3; (C) c-Myc, survivin and cyclin D1. C (white column), control; 80 (black column), treated with $80 \mathrm{mg} / \mathrm{kg}$ of STX-0119; 160 (hatched column), treated with $160 \mathrm{mg} / \mathrm{kg}$ of STX-0119.

\section{A}
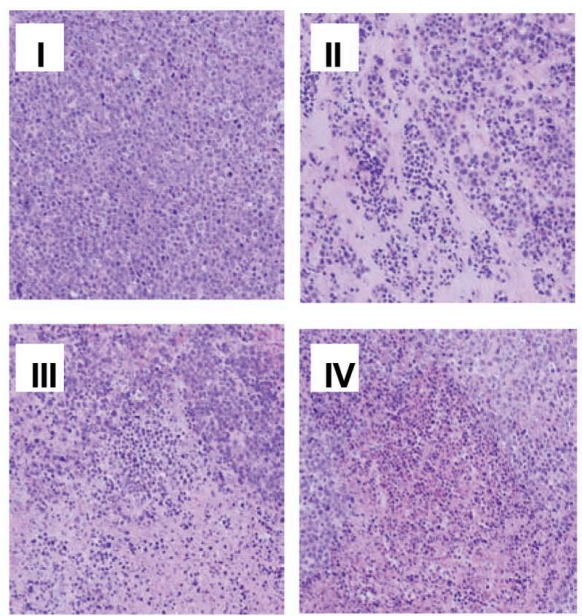

B c-myc

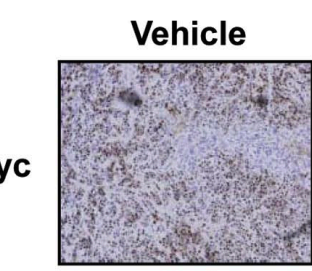

Ki67

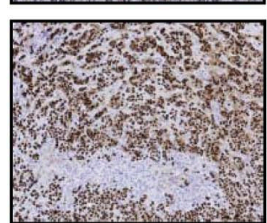

pSTAT3

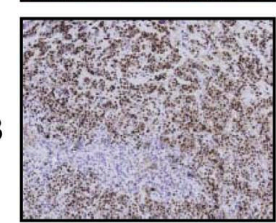

STAT3

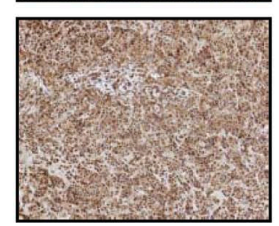

STX-0119

$160 \mathrm{mg} / \mathrm{kg}$
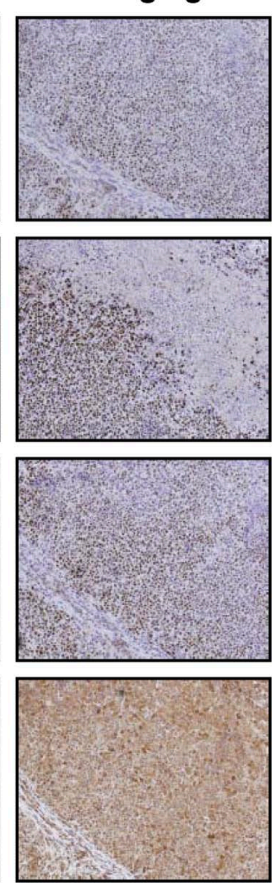

Figure 8. IHC analysis of SCC3 tumors treated with STX-0119. (A) H\&E staining of tumors treated with $160 \mathrm{mg} / \mathrm{kg}$ STX-0119. I, control (untreated); II, 7 days after STX-0119 administration; III, 14 days; IV, 21 days. (B) Down-regulation of c-myc and Ki67 proteins in STX-0119-treated tumors. SCC3 tumors were resected on day 21 after the start of STX-0119 administration $(160 \mathrm{mg} / \mathrm{kg})$. Immunostaining using antibodies against STAT3, phosphorylated STAT3, c-myc and Ki67 was performed. Magnification, x100.

expression of STAT3 and phosphorylated STAT3 was maintained before and after the treatment. Downstream target genes like c-myc, cyclin D1 and survivin apeared to be down-regulated in the late phase, particularly in the tumors treated with $160 \mathrm{mg}$ / $\mathrm{kg}$ of STX-0119 (Fig. 7). Similar to the in vitro experiments, the up-regulation of cleaved caspase-3 expression was noted in the tumors in the late phase (from day 14) of STX-0119 treatment.

IHC analysis of SCC-3 tumors treated with STX-0119. Seven days after the start of STX-0119 administration, morpho- 
logical change was noted suggesting a cytotoxic effect. From day 13, cell infiltration with necrosis occurred (Fig. 8A). In viable tumors, most cells were positive for STAT3; however Ki67- and c-myc-positive tumor cells appeared to decrease in number compared to the control (Fig. 8B).

\section{Discussion}

STAT3 is a member of a family of DNA-binding molecules, which regulate numerous physiological and oncogenic signaling pathways leading to the induction of target gene expression through STAT3-SH2 dimerization and phosphorylation, and to the promotion of cell proliferation and induction of anti-apoptotic activity and angiogenesis.

Recent studies have showed that STAT3 is constitutively activated in various types of cancers including hematological and solid cancers. Evidence has demonstrated that persistent STAT3 activation may impair immune responses by enhancing immunological tolerance and inducing immunological evasion in the tumor site. Additionally, aberrant STAT3 activation plays an important role in establishing loops of autocrine and/ or paracrine cytokines, particularly IL-6, which may partly contribute to tumorigenesis in some types of cancers $(16,17)$.

Experimental approaches to blocking STAT3 signaling using small interfering RNA (siRNA), small hairpin RNA (shRNA) and STAT3 antisense have been successful in inhibiting cell proliferation in vitro (18) and tumor growth in vivo (19). However, STAT3 silencing has been reported to have no toxic effect on normal cells (20). These observations suggest that STAT3 may be a potential target in cancer therapy.

Previously, we identified a novel small-molecule inhibitor of STAT3 dimerization, STX-0119, using biochemical and virtual screening methods (STAT3-dependent luciferase reporter gene assay and STAT3 dimerization assay using FRET) for antagonizing the activity of STAT3-SH2 binding (15). The crystal structure of a STAT3-based computer model (in silico) obtained with a docking simulation algorithm named Consensus-Dock revealed that STX-0119 binds directly to the $\mathrm{SH} 2$ region of the STAT3 monomer and antagonizes the dimerization.

In the present study, we investigated the antitumor activity of STX-0119 in vitro and in vivo and investigated the mechanism involved through biochemical and biological assays. Our study revealed that STX-0119 had a potent growth-inhibitory effect on the human lymphoma cell line SCC3 (STAT3activated), and effectively down-regulated downstream target genes of the STAT3 signaling pathway including c-myc, Bcl-xL, cyclin D1 and survivin without significantly affecting STAT3 phosphorylation. Matsuno et al (15) reported similar results from experiments in vitro using human breast cancer MDA-MB-468 cells. More impressively, STX-0119 exhibited stronger growth inhibition against SCC3 tumors in vivo than did AG490.

A notable observation in vivo was the two-phased effect of STX-0119 against SCC3 tumors: rapid inhibition at approximately day 4 (early) and sustained inhibition after day 21 (late). Biochemical analysis using resected tumors treated with STX-0119 demonstrated extensive apoptosis of the tumor in the late phase; however there may be some inhibitory effect on cancer stem cells leading to late-phase inhibition.
Recently, Sherry et al (21) demonstrated STAT3-dependent proliferation and maintenance in glioblastoma stem cells, which can be inhibited by a STAT3 signaling inhibitor. These results suggest cancer stem cells to be a novel target for smallmolecule STAT3 signaling inhibitors.

Recently, many JAK-STAT3 inhibitors, which down-regulate STAT3 phosphorylation (Tyr-705) and induce apoptosis in cancer cells by inhibiting STAT3 signaling, have been developed, including STAT3 DNA decoy (22), LLL-3 (23), indirubin derivative E804 (24), AG490 (25), WP1066 (26), FLLL31 and 32 (27), cucurbitacin analogue (28), SD1029 (29), JSI-124 (30) and the JAK2 inhibitor AZD1480 (31). Only a few compounds showed antitumor activity in vivo; however AZD1480 exhibited very strong antitumor activity against solid tumors like breast and prostate cancer in vitro $\left(\mathrm{IC}_{50}<0.1 \mu \mathrm{M}\right)$ and in vivo compared to other compounds. By contrast, only a few inhibitors of STAT3-SH2 dimerization have been reported, namely peptide mimetics (32), STA-21 (33), Stattic (34), S3I-201 (NSC74859) (35) and 5,15-DPP (36). Based on these findings, STX-0119 showed the strongest antitumor activity, particularly in vivo, among the STAT3 dimerization inhibitors. Notably, STAT3 phosphorylation was suppressed by Static and S3I-201, but not STA-21 and STX-0119. As STX-0119 was demonstrated to regulate STAT3 transcriptional activity and induce apoptosis in cancer cells, there may be different mechanisms affecting the phosphorylation or translocation of STAT3 into the nucleus. In the present study, we obtained evidence that STX-0119 inhibited phosphorylated STAT3 in the cytosol, but not STAT3 in the nucleus, using a subcellular fractionation kit and WB (Fig. 5). These results suggest mainly two possibilities: i) the inhibition of STX-0119 activity against dimerization and translocation of STAT3 leading to the breakdown of phosphorylated STAT3 in the cytosol, or ii) no significant effect of STX-0119 on transported phosphorylated STAT3 in the nucleus. In contrast, AG490 significantly suppressed STAT3 phosphorylation in both the cytosol and nucleus. Seemingly, there may be a different mechanism of activity between the dimerization inhibitor, STX-0119, and the kinase inhibitor, AG490. To investigate the precise mechanism of STX-0119 activity, a time-course study of phosphorylated STAT3 localization is warranted.

Finally, one important issue which requires investigation is the fact that the antitumor efficacy in nude mice was weak compared to that of STAT3 signaling inhibitors such as AZD1480. Considering the high concentration of STX-0119 in mouse plasma after an oral administration in a pharmacokinetic study reported previously (15), there may be a discrepancy between the plasma concentration and antitumor effect. Poor uptake of STX-0119 in the tumor site and a weak immunological antitumor effect in nude mice triggered by overcoming the STAT3-mediated immunosuppression induced in the tumor microenvironment (37) may be responsible for this discrepancy. In the future, intratumoral pharmacokinetics and immunocompetent mice should be utilized.

In conclusion, we identified a novel small-molecule inhibitor of STAT3 dimerization using biochemical and virtual screening methods and demonstrated that it shows a potent antitumor effect in vitro and in vivo against constitutively 
STAT3-activated cancer cells. These observations suggest STX-0119 to be a promising candidate for STAT3-signaling therapeutics in STAT3-activated hematological malignancies.

\section{Acknowledgements}

This work was supported by a grant from the National Institute of Biomedical Innovation, Japan (06-2).

\section{References}

1. Zhong Z, Wen L and Darnell JE Jr: Stat3 and Stat4: members of the family of signal transducers and activators of transcription. Proc Natl Acad Sci USA 91: 4806-4810, 1994.

2. Bromberg J and Darnell JE Jr: The role of STATs in transcriptional control and their impact on cellular functions. Oncogene 19: 2468-2473, 2000.

3. Shen Y, Schlessinger K, Zhu X, Meffre E, Quimby F, Levy DE and Darnell JE Jr: Essential role of STAT3 in postnatal survival and growth revealed by mice lacking STAT3 serine 727 phosphorylation. Mol Cell Biol 21: 407-419, 2004.

4. Bhattacharya S, Ray RM and Johnson LR: STAT3-mediated transcription of Bcl-2, Mcl-1 and c-IAP2 prevents apoptosis in polyamine-depleted cells. Biochem J 392: 335-344, 2005.

5. Kiuchi N, Nakajima K, Ichiba M, Fukuda T, Narimatsu M, Mizuno K and Hirano T: STAT3 is required for the gp130mediated full activation of the c-myc gene. J Exp Med 189: 63-73, 1999.

6. Bromberg JF, Wrzeszczynska MH, Devgan G, Zhao Y, Pestell RG, Albanese C and Darnell JE Jr: Stat 3 as an oncogene. Cell 98: 295-303, 1999.

7. Leslie K, Lang C, Devgan G, Azare J, Beridhaj M, Gerald W, Kim YB, Paz K, Darnell JE, Albanese C, Sakamaki T, Pestell R and Bromberg J: Cyclin D1 is transcriptionally regulated by and required for transformation by activated signal transducer and activator of transcription 3. Cancer Res 66: 2544-2552, 2006

8. Niu G, Wright KL, Huang M, Song L, Haura E, Turkson J, Zhang S, Wang T, Sinibaldi D, Coppola D, Heller R, Ellis LM, Karras J, Bromberg J, Pardoll D, Jove R and Yu HD: Constitutive Stat3 activity up-regulates VEGF expression and tumor angiogenesis. Oncogene 27: 2000-2008, 2002.

9. Konnikoval L, Simeon MC, Kruger MM, Kotecki M and Cochran BH: Signal transducer and activator of transcription 3 (STAT3) regulates human telomerase reverse transcriptase (hTERT) expression in human cancer and primary cells. Cancer Res 65: 6516-6520, 2005.

10. Kim DJ, Tremblay ML and Digiovanni J: Protein tyrosinase phosphatase, TC-PTP, SHP1, and SHP2, cooperate in rapid dephosphorylation of Stat 3 in keratinocytes following UVB irradiation. PloS One 5: e10290, 2010.

11. Groner B, Lucks P and Borghouts C: The function of Stat3 in tumor cells and their microenvironment. Semin Cell Dev Biol 19: 341-350, 2008

12. Bowman T, Garcia R, Turkson J and Jove R: STATs in oncogenesis. Oncogene 19: 2474-2488, 2000.

13. Turkuson $\mathbf{J}$ and Jove R: STAT proteins: novel molecular targets for cancer drug discovery. Oncogene 19: 6613-6626, 2000.

14. Siddiquee K and Turkson J: STAT3 as a target for inducing apoptosis in solid and hematological tumors. Cell Res 18: 254-267, 2008

15. Matsuno K, Masuda Y, Uehara Y, Sato H, Muroya A, Takahashi O, Yokotagawa T, Furuya T, Okawara T, Otsuka M, Ogo N, Ashizawa T, Oshita C, Tai S, Ishii H, Akiyama Y and Asai A: Identification of a new series of STAT3 inhibitors by virtual screening. ACS Med Chem Lett 1: 371-375, 2010.

16. Culing Z, Steiner H, Bartsch G and Hobisch A: Interleukin-6 regulation of prostate cancer cell growth. J Cell Biochem 95: 497-505, 2005.

17. Grivennikov S and Karin M: Autocrine IL-6 signaling: a key event in tumorigenesis? Cancer Cell 13: 7-9, 2008.

18. Gao L, Zhang L, Hu J, Li F, Shao Y, Zhao D, Kalvakolanu DV, Kopecko DJ, Zhao X and Xu DQ: Down-regulation of signal transducer and activator of transcription 3 expression using vector-based small interfering RNAs suppresses growth of human prostate tumors in vivo. Clin Cancer Res 11: 6333-6341, 2005.
19. Ling $X$ and Arlinghaus RB: Knockdown of STAT3 expression by RNA interference inhibits the induction of breast tumors in immunocompetent mice. Cancer Res 65: 2532-2536, 2005.

20. Buettner R, Mora LB and Jove R: Activated STAT signaling in human tumors provides novel molecular targets for therapeutic intervention. Clin Cancer Res 8: 945-954, 2002.

21. Sherry MM, Reeves A, Wu JK and Cochran BH: STAT3 is required for proliferation and maintenance of multipotency in glioblastoma stem cells. Stem Cells 27: 2383-2392, 2009.

22. Lui VW, Boehm AL, Koppikar P, Leeman RJ, Johnson D, Ogagan M, Childs E, Freilino M and Grandis JR: Antiproliferative mechanisms of a transcription factor decoy targeting signal transducer and activator of transcription (STAT) 3: the role of STAT1. Mol Pharmacol 71: 1435-1443, 2007.

23. Fuh B, Sobo M, Cen L, Josiah D, Hutzen B, Cisek K, Bhasin D, Regan N, Lin L, Chan C, Caldas H, DeAngelis S, Li C, Li PK and Lin J: LLL-3 inhibits STAT3 activity, suppresses glioblastoma cell growth and prolongs survival in a mouse glioblastoma model. Br J Cancer 100: 106-112, 2009.

24. Nam SBR, Turkson J, Kim D, Cheng JQ, Muehlbeyer S, Hippe F, Vatter S, Merz KH, Eisenbrand G and Jove R: Indirubin derivatives inhibit Stat 3 signaling and induce apoptosis in human cancer cells. Proc Natl Acad Sci USA 102: 5998-6003, 2005.

25. Spiekermann K, Biethahn S, Wilde S, Hiddemann W and Alves F: Constitutive activation of STAT transcription factors in acute myelogenous leukemia. Eur J Haematol 67: 63-71, 2001.

26. Iwamaru A, Szymanski S, Iwado E, Aoki H, Yokokawa T, Fokt I, Hess K, Conrad C, Madden T, Sawaya R, Kondo S, Priebe W and Kondo Y: A novel inhibitor of the STAT3 pathway induces apoptosis in malignant glioma cells both in vitro and in vivo. Oncogene 26: 2435-2444, 2007.

27. Lin L, Hutzen B, Zuo M, Ball S, Deangelis S, Foust E, Pandit B, Ihnat MA, Shenoy SS, Kulp S, Li PK, Li C, Fuchs J and Lin J: Novel STAT3 phosphorylation inhibitors exhibit potent growthsuppressive activity in pancreatic and breast cancer cells. Cancer Res 70: 2445-2454, 2010.

28. Sun J, Blaskovich M, Jove R, Livingston S, Coppola D and Sebti S: Cucurbitacin Q: a selective STAT3 activation inhibitor with potent antitumor activity. Oncogene 24: 3236-3245, 2005.

29. Duan Z, Bradner JE, Greenberg E, Levine R, Foster R, Mahoney J and Seiden MV: SD-1029 inhibits signal transducer and activator of transcription 3 nuclear translocation. Clin Cancer Res 12: 6844-6852, 2006.

30. Blaskovich MA, Sun J, Cantor A, Turkson J, Jove R and Sebti SM: Discovery of JSI-124 (Cucubitacin I), a selective Janus kinase/signal transducer and activator of transcription 3 signaling pathway inhibitor with potent antitumor activity against human and murine cancer cells in mice. Cancer Res 63 : 1270-1279, 2003.

31. Hedvat M, Huszar D, Herrmann A, Gozgit JM, Schroeder A, Sheehy A, Buettner R, Proia D, Kowolik CM, Xin H, Armstrong B, Bebernitz G, Weng S, Wang L, Ye M, McEachern K, Chen H, Morosini D, Bell K, Alimzhanov M, Loannidis S, McCoon P, Cao ZA, Yu H, Jove R and Zinda M: The JAK2 inhibitor AZD1480 potently blocks stat 3 signaling and oncogenesis in solid tumors. Cancer Cell 16: 487-497, 2009.

32. Mandal PK, Limbrick D, Coleman DR IV, Dyer GA, Ren Z, Birtwistle JS, Xiong C, Chen X, Briggs JM and McMurray JS: Conformationally constrained peptidomimetic inhibitors of signal transducer and activator of transcription 3: evaluation and molecular modeling. J Med Chem 52: 2429-2442, 2009.

33. Song H, Wang R, Wang S and Lin J: A low-molecular-weight compound discovered through virtual database screening inhibits stat 3 function in breast cancer cells. Proc Natl Acad Sci USA 102: 4700-4705, 2005.

34. Schust J, Sperl B, Hollis A, Mayer TU and Berg T: Stattic: a small-molecule inhibitor of STAT3 activation and dimerization. Chem Biol 13: 1235-1242, 2006.

35. Siddiquee K, Zhang S, Guida W, Blaskovich MA, Greedy B, Lawrence HR, Yip ML, Jove R, McLaughlin MM, Lawrence NJ, Sebti SM and Turkson J: Selective chemical probe inhibitor of Stat3, identified through structure-based virtual screening induces antitumor activity. Proc Natl Acad Sci USA 104: 7391-7396, 2007.

36. Uehara Y, Mochizuki M, Matsuno K, Haino T and Asai A: Novel high-throughput screening system for identifying STAT3-SH2 antagonists. Biochem Biophys Res Commun 380: 627-631, 2009.

37. Yu H, Kortylewski M and Pardoll D: Crosstalk between cancer and immune cells: role of STAT3 in the tumor microenvironment. Nat Rev Immunol 7: 41-51, 2007. 